ESAIM: Proceedings, Vol. 4, 1998, 59-72

Contrôle et Équations Aux DÉRivées Partielles

http://www.emath.fr/proc/Vol.4/

\title{
SINGULARITIES OF THE MINIMUM TIME FUNCTION FOR SEMILINEAR PARABOLIC SYSTEMS
}

\author{
PAOLO ALBANO ${ }^{1}$ \\ Dipartimento di Matematica, Università di Roma "Tor Vergata" \\ Via della Ricerca Scientifica, 00133 Roma, Italy \\ Fax: +39-6-72594699 \\ Electronic mail: albano@mat.utovrm.it \\ PIERMARCO CANNARSA ${ }^{1}$ \\ Dipartimento di Matematica, Università di Roma "Tor Vergata" \\ Via della Ricerca Scientifica, 00133 Roma, Italy \\ Fax: +39-6-72594699 \\ Electronic mail: cannarsa@mat.utovrm.it
}

Key Words: minimum time problem, parabolic equations, optimality conditions.

AMS Subject Qualification : 49J20, 49M29.

\footnotetext{
${ }^{1}$ Partially supported by the Italian National Project MURST $40 \%$ "Problemi nonlineari...".

Article published by EDP Sciences and available at http://www.edpsciences.org/proc or http://dx.doi.org/10.1051/proc:1998020
} 


\begin{abstract}
The time optimal control problem for a semilinear parabolic systems is here studied. The singular points of the Minimum Time function are characterized as the initial point of multiple time-optimal trajectories. Moreover, an example is given to show how such singularities may develop.
\end{abstract}




\section{Introduction}

Let $\Omega \subset \mathbb{R}^{n}$ be open and bounded with smooth boundary and let $N \in \mathbb{N}$. Let us consider the controlled parabolic system

$$
\left\{\begin{array}{l}
\frac{\partial y}{\partial t}(t, \xi)=\Delta_{\xi} y(t, \xi)+f(y(t, \xi))+u(t, \xi), \quad t>0 \\
y(0, \xi)=x(\xi), \quad \xi \in \Omega
\end{array}\right.
$$

where $x \in L^{2}\left(\Omega ; \mathbb{R}^{N}\right)$, i.e. $x: \Omega \rightarrow \mathbb{R}^{N}$ is a vector-valued function with square integrable components, and $u:\left[0, \infty\left[\times \Omega \rightarrow \mathbb{R}^{N}\right.\right.$ — the control strategy — is a measurable function such that

$$
\int_{\Omega}|u(t, \xi)|^{2} d \xi \leq R^{2}
$$

for all $t \geq 0$ and some constant $R>0$. Moreover, the nonlinear term $f: \mathbb{R}^{N} \rightarrow$ $\mathbb{R}^{N}$ is a function of class $C^{1}$ such that, for suitable constants $L_{0}, L_{1}>0$,

$$
\begin{array}{r}
f(0)=0 \\
|\nabla f(\xi)| \leq L_{0} \\
|\nabla f(\xi)-\nabla f(\eta)| \leq L_{1}|\xi-\eta|
\end{array}
$$

for all $\xi, \eta \in \mathbb{R}^{N}$.

We are interested in the Time Optimal Control Problem for the above system, which consists of finding the control strategies $u$ that steer (1) to a closed target $K$ in minimum time.

In this paper, we will take $K$ to be a closed ball in $L^{2}\left(\Omega ; \mathbb{R}^{N}\right)$ with radius $r>0$ and center at 0 , i.e.

$$
B_{r}=3\{x \in H:\|x\| \leq r\} .
$$

Our approach may, however, be easily generalized to target that satisfy and "interior sphere condition".

The history of this problem dates back to the works [15], [16], [17], [18], [3], [14], [19] (see also the book [21]), where it was studied for linear systems $(f \equiv 0)$ using the Pontryagin Maximum Principle. The linear problem is also considered in [9] from the point of view of Dynamic Programming. Nonlinear time optimal control problems in infinite dimensions were treated in [4], [5], [12], [13] following the Dynamic Programming approach. A comprehensive description of many of these results is given in the book [20].

In this paper we will focus our attention on some special aspects of nonlinear minimum time problems. In order to explain the results we have in mind, it is convenient to rewrite system (1) in abstract form, that is

$$
\left\{\begin{array}{l}
y^{\prime}(t)=A y(t)+F(y(t))+u(t), \quad t>0, \\
y(0)=x \in H
\end{array}\right.
$$

ESAIM: Proc., Vol. 4, 1998, 59-72 
Here and in the sequel we denote by $H$ the Hilbert space $L^{2}\left(\Omega ; \mathbb{R}^{N}\right)$ with the usual scalar product $\langle\cdot, \cdot\rangle$ and norm $\|\cdot\|$. Moreover, $A: D(A) \subset H \rightarrow H$ is the unbounded linear operator defined by

$$
\begin{array}{r}
D(A)=H_{0}^{1}\left(\Omega ; \mathbb{R}^{N}\right) \cap H^{2}\left(\Omega ; \mathbb{R}^{N}\right) \\
A x=\Delta x, \quad \forall x \in D(A),
\end{array}
$$

and $F: H \rightarrow H$ is the nonlinear map

$$
F(x)(\xi)=f(x(\xi)), \quad \xi \in \Omega .
$$

It is well known that $A$ is the generator of an analytic semigroup of contractions in $H$, and that there exists $\omega>0$ such that

$$
\langle A x, x\rangle \leq-\omega\|x\|^{2} \text { for all } x \in D(A) .
$$

A control strategy for system (3) is then a measurable function $u:[0, \infty[\rightarrow H$ such that $\|u(t)\| \leq R$ for a.e. $t \geq 0$, where $R$ is a given positive number. We will denote by $\mathcal{U}$ the set of all control strategies.

In view of assumption (2), $F$ is a Lipschitz map in $H$. So, for any initial condition $x \in H$ and any control strategy $u$, state equation (3) has a unique mild solution that will be denoted by $y^{x, u}(t)$.

We now proceed to recast in abstract terms the time optimal control problem

that we introduced before. Let $r>0$ be given, and set

$$
\tau(x, u)=\inf \left\{t \geq 0:\left\|y^{x, u}(t)\right\| \leq r\right\}
$$

for any $x \in H$ and any control strategy $u$. Then, $\tau(x, u) \in[0,+\infty]$, and we note that $\tau(x, u)$ is the time taken by the trajectory $y^{x, u}(t)$ to reach target $B_{r}$.

We define the controllable set $\mathcal{R}$ to be the set of all points $x \in H$ such that $\tau(x, u)<+\infty$ for some $u$. In general, the structure of $\mathcal{R}$ can be very irregular. However, under the controllability assumption

$$
\left(L_{0}-\omega\right) r<R
$$

the controllable set $\mathcal{R}$ is an open neighbourood of the target $B_{r}$.

The value function of the above problem is called the minimum time function, and is defined as

$$
T: \mathcal{R} \rightarrow\left[0,+\infty\left[, \quad T(x):=\inf _{u \in \mathcal{U}} \tau(x, u) .\right.\right.
$$

The basic idea of the Dynamic Programming method consists of using the value function to derive optimality conditions for problem (3)-(8). For Linear Quadratic problems this approach is very powerful as such a function is smooth, provides the optimal synthesis, and can be determined by solving the Riccati equation, see [6].

For nonlinear problems the situation is much more complicated. Indeed, the value function is no longer differentiable, and the Riccati equation has to be 
replaced by the Hamilton-Jacobi equation, which, in the case of the above time optimal control problem, has the form

$$
R\|D T(x)\|-\langle A x+F(x), D T(x)\rangle=1, \quad x \in \mathcal{R} .
$$

However, useful optimality conditions can still be derived from properties of the value function. Results in this direction were obtained in [7], [8] for the Mayer optimal control problem. For the minimum time problem an analogous approach is developed in [2]. In particular, it is shown in [2] that the points $x \in$ $\mathcal{R}$ at which $T$ is differentiable are exactly the points for which the optimal control problem (3)-(8) admits a unique optimal trajectory. This result, combined with the differentiability of $T$ obtained in [9] for the case of $F=0$, yields the uniqueness of optimal traject ories for the linear problem, a known result that was proved in [17]. In section 2 of this paper we recall part of the results of [2] and apply them to system (1).

In light of the above considerations, it is clear that one of the main differences between the nonlinear problem (3)-(8) and its linearized version, is the fact that $T$ may develop singularities when $F$ is not identically 0 . Such a phenomenon is well known for finite dimensional control problems, and so, in particular, for abstract problems of the form (3)-(8), not necessarily related to the optimal control of partial differential equations. Indeed, the distance from a closed set, that is a special example of minimum time function, may well be singular at some point.

However, the open question remains whether the minimum time function associated with a system of the form (1) may still be nonsmooth. In section 2 of this paper we provide such an example for a $2 \times 2$ reaction diffusion system.

Finally, we would like to note that we have chosen to treat a special system like (1) just for simplicity. Most of the results we will describe in this paper apply, without any change, to more general parabolic systems associated of the form

$$
A x(\xi)=\sum_{i, j=1}^{n} A_{i j}(\xi) \frac{\partial^{2} x}{\partial \xi_{i} \partial \xi_{j}}(\xi)+\sum_{i=1}^{n} B_{i}(\xi) \frac{\partial x}{\partial \xi_{i}}(\xi)+C(\xi)=x(\xi), \quad \xi \in \Omega,
$$

under the Legendre-Hadamard ellipticity condition

$$
\sum_{i, j=1}^{n} \eta_{i} \eta_{j}\left(A_{i j}(\xi) \zeta \mid \zeta\right) \geq \nu|\eta|^{2}|\zeta|^{2}, \quad \forall \xi \in \Omega,
$$

for all vectors $\eta \in \mathbb{R}^{n}, \zeta \in \mathbb{R}^{N}$, and some constant $\nu>0$. The corresponding results ensuring the generation of an analytic semigroup in $H$ are proved in [1] (see also [10])

\section{Singularities of $T$}

In this section we will study the optimal control problem (3)-(8) where $A$ and $F$ are defined in (4) and (5), respectively. Moreover, we suppose that assumptions (2) and (7) hold throughout. We warn the reader that this restriction 
is made just to simplify the exposition. All the results we describe in this section hold for more general time optimal control problems in infinite dimensions, provided that $A$ and $F$ satisfy suitable abstract conditions. In particular, $A$ must be the generator of an analytic semigroup, $e^{t A}$, of compact operators (for $t>0)$ in $H$, and $F: H \rightarrow H$ must be Lipschitz continuous and differentiable along the directions of a suitable subspace of $H$. We refer the interested reader to [2] for all these extensions.

First of all we note that the existence of optimal controls for problem (3)(8) follows from the compactness of $e^{t A}$ (for $t>0$ ). We give the proof for the reader's convenience.

Proposition 2.1 Let $x$ be an element of $\mathcal{R}$. Then there exists an optimal control $u$, i.e. $u \in \mathcal{U}$ such that $\left\|y^{x, u}(T(x))\right\|=r$.

Proof - Let $x \in \mathcal{R} \backslash B_{r}$ and let $u_{n} \in \mathcal{U}$ be such that

$$
\tau\left(x, u_{n}\right) \rightarrow T(x) .
$$

From the definition of $\tau(x, \cdot)$ it follows that

$$
\left\|y^{x, u_{n}}\left(\tau\left(x, u_{n}\right)\right)\right\|=r .
$$

By passing to a subsequence we can assume that

$$
u_{n} \rightarrow u_{\infty}, \quad \text { as } n \rightarrow \infty
$$

where the convergence is weak $-*$ in $L^{\infty}\left(0, T_{*} ; B_{R}\right)$, and $T_{*}$ is chosen so that $\tau\left(x, u_{n}\right) \leq T_{*}$ for all sufficiently large $n$. By the compactness of $e^{t A}$ we obtain

$$
\int_{0}^{t} e^{(t-s) A} u_{n}(s) d s \rightarrow \int_{0}^{t} e^{(t-s) A} u_{\infty}(s) d s \quad \text { as } n \rightarrow \infty
$$

for any $t>0$, where the convergence is strong in $H$. Then, passing to the limit in (10) we conclude that

$$
r=\lim _{n \rightarrow \infty} \| y^{x, u_{n}}\left(\tau\left(x, u_{n}\right)\|=\| y^{x, u_{\infty}}(T(x)) \| .\right.
$$

Definition 2.2 We say that a point $x \in H$ is singular for the Minimum Time Function $T$, or, equivalently, that $T$ has a singularity at $x$, if $T$ is not Fréchet differentiable at $x$.

The singular points of $T$ can be characterized in terms of optimal controls, as stated below.

Theorem 2.3 A point $x \in H$ is singular for the $T$ if and only if there exist at least two time optimal trajectories for system (3) with initial point $x$.

We give a sketch of the proof of the above theorem. See [2] for details. Proof - We have to show that (i) $\Leftrightarrow$ (ii) where 
(i) $T$ is differentiable at $x$;

(ii) there exists a unique time optimal trajectory with initial point $x$.

The following steps are the key points of our argument.

Step 1: Pontryagin Maximum Principle and Co-State Inclusion.

Let $x \in \mathcal{R}$ be fixed, and let $u(t)$ be an optimal control at $x$. We denote by $y(t):=y^{x, u}(t)$ the related optimal trajectory, and we set

$$
T^{*}=T(x) \text {. }
$$

Let $p(t)$ be the corresponding co-state, i. e. the solution of the adjoint system

$$
\left\{\begin{array}{l}
p^{\prime}(t)=-A^{*} p(t)-\Phi(t) p(t), \quad t>0, \\
p\left(T^{*}\right)=\frac{y\left(T^{*}\right)}{r\left[R-r\left(L_{0}-\omega\right)\right]}
\end{array}\right.
$$

Here $\Phi(t) \in \mathcal{L}(H)$ is defined as

$$
(\Phi(t) z)(\xi)=[D f]^{*}(y(t, \xi)) z(\xi), \quad \xi \in \Omega, z \in H,
$$

where $f$ is the function that generates $F$ by (5), and $[D f]^{*}$ denotes the transpose of matrix $D f$.

Then, by the Pontryagin Maximum Principle we have that $u$ is related to $p$ by the formula

$$
u(t)=r \frac{p(t)}{\|p(t)\|} \quad \text { a.e. } t \in\left[0, T^{*}\right] .
$$

Moreover, the following inclusion is proved in [2]:

$$
p(t) \in D^{+} T(y(t)), \quad \forall t \in\left[0, T^{*}[,\right.
$$

where the superdifferential $D^{+} T$ is defined by

$$
D^{+} T\left(z_{0}\right)=\left\{p \in H: \limsup _{z \rightarrow z_{0}} \frac{T(z)-T\left(z_{0}\right)-\left\langle p, z-z_{0}\right\rangle}{\left\|z-z_{0}\right\|} \leq 0\right\}, \quad \forall z_{0} \in H .
$$

STEP 2: (i) $\Rightarrow$ (ii).

Let $x, u, y, p$ and $T^{*}$ be defined as above. Then, by (11) and (12) we have that

$$
\left\{\begin{array}{l}
y^{\prime}(t)=A y(t)+F(y(t))+r \frac{p(t)}{\|p(t)\|} \\
p^{\prime}(t)=-A^{*} p(t)-\Phi(t) p(t)
\end{array}\right.
$$

for all $t \in] 0, T^{*}[$, with initial conditions

$$
\begin{array}{r}
y(0)=x \\
p(0)=D T(x)
\end{array}
$$

ESAIM: Proc., Vol. 4, 1998, 59-72 
Notice that (15) follows from (13) and the fact that $D^{+} T(x)=\{D T(x)\}$. Now, following an argument of [11], one can obtain a uniqueness result for problem $(14)(15)$. Therefore, the pair $(y, p)$ is unique.

\section{Step 3: Semiconcavity of $T$.}

It is proved in [2] that the Minimum Time Function is semiconcave in $\mathcal{R} \backslash B_{r}$, that is $T$ can be represented, locally, as the sum of a concave function plus a quadratic function. Consequently, $D^{+} T(x)$ is a nonempty set for any $x \in \mathcal{R} \backslash B_{r}$.

Therefore, $T$ is differentiable at a point $x$ if and only if $D^{-} T(x)$ is also nonempty, where

$$
D^{-} T(x)=\left\{p \in H: \liminf _{x^{\prime} \rightarrow x} \frac{T\left(x^{\prime}\right)-T(x)-\left\langle p, x^{\prime}-x\right\rangle}{\left\|x^{\prime}-x\right\|} \geq 0\right\} .
$$

is the subdifferential of $T$ at $x$.

STEP 4: (ii) $\Rightarrow$ (i).

In light of the previous step, we only need to show that

$$
p(t) \in D^{-} T(y(t)), \quad \forall t \in\left[0, T^{*}[.\right.
$$

Moreover, it sufficies to prove (16) in the case of $t=0$. For this purpose, let us consider a sequence $x_{k} \rightarrow x$ as $k \rightarrow \infty$ such that

$$
\liminf _{x^{\prime} \rightarrow x} \frac{T\left(x^{\prime}\right)-T(x)-\left\langle p(0), x^{\prime}-x\right\rangle}{\left\|x^{\prime}-x\right\|}=\lim _{k \rightarrow \infty} \frac{T\left(x_{k}\right)-T(x)-\left\langle p(0), x_{k}-x\right\rangle}{\left\|x_{k}-x\right\|} .
$$

Let now $y_{k}(\cdot)$ be optimal trajectories at $x_{k}$ and let $p_{k}(\cdot)$ be the solutions of (11) corresponding to $y_{k}(\cdot)$. Passing to a subsequence, assumption (ii) implies that $y_{k}(\cdot)$ converges uniformly to $y(\cdot)$. Then, recalling (11) one can prove that $p_{k}(\cdot) \rightarrow p(\cdot)$ as $k \rightarrow \infty$. Therefore we have that

$$
\begin{aligned}
T\left(x_{k}\right)-T(x) & -\left\langle p(0), x_{k}-x\right\rangle \\
& =T\left(x_{k}\right)-T(x)+\left\langle p_{k}(0), x-x_{k}\right\rangle-\left\langle p(0)-p_{k}(0),-x+x_{k}\right\rangle \\
& \geq-C_{R}\left\|x_{k}-x\right\|^{2}-\left\|p(0)-p_{k}(0)\right\| \cdot\left\|x_{k}-x\right\| .
\end{aligned}
$$

Hence,

$$
\lim _{k \rightarrow \infty} \frac{T\left(x_{k}\right)-T(x)-\left\langle p(0), x_{k}-x\right\rangle}{\left\|x_{k}-x\right\|} \geq 0
$$

and the proof is complete.

\section{Examples}

The purpose of this section is to give an example of a system of type (1) such that the singular set of the associated minimum time function is nonempty. In light of Theorem 2.3 it suffices to provide an example with multiple timeoptimal trajectories. Following this idea, we first construct such an example with a nonsmooth term $f$ and then we regularize this term to have assumption (2) satisfied. 
Example 3.1 Let $\Omega=(0, \pi), N=2$ and $R=1$, the target radius $r>0$ to be fixed later. Consider the parabolic system in $(0,+\infty) \times(0, \pi)$

$$
\left\{\begin{array}{l}
\frac{\partial y_{1}}{\partial t}-\frac{\partial^{2} y_{1}}{\partial \xi^{2}}=-\left|y_{2}\right|+u_{1} \\
\frac{\partial y_{2}}{\partial t}-\frac{\partial^{2} y_{2}}{\partial \xi^{2}}=u_{2}
\end{array}\right.
$$

with homogeneous Dirichlet boundary conditions

$$
y(t, 0)=y(t, \pi)=0, \quad t>0 .
$$

Let us impose the initial condition $y(0, \cdot)=x^{\rho}$, where

$$
x_{1}^{\rho}(\xi)=\rho \sqrt{\frac{2}{\pi}} \sin \xi, \quad x_{2}^{\rho}(\xi)=0, \quad \forall \xi \in(0, \pi)
$$

and $\rho>r$ will be fixed later. Notice that $x^{\rho} \notin B_{r}$. We note that the nonlinear term of system (17) does not satisfy the smoothness assumption (2). On the other hand, the absolute value is Lipschitz with constant $L_{0}=1$, and $\omega=1$ in the above example. Therefore, assumption (7) is "morally" satisfied for any $r>0$.

Let us compute first the minimum time, $t_{0}=t_{0}\left(x^{\rho}\right)$, needed to steer system (17) to $B_{r}$ under the state constraint

$$
y_{2} \equiv 0 \text {. }
$$

Using the Fourier expansion of $y_{1}$ and $u_{1}$

$$
y_{1}(t, \xi)=\sum_{k=1}^{\infty} \alpha_{k}(t) \sin (k \xi), \quad u_{1}(t, \xi)=\sum_{k=1}^{\infty} \lambda_{k}(t) \sin (k \xi),
$$

from (17) and (19) we obtain

$$
\begin{array}{r}
\sum_{k=1}^{\infty}\left[\alpha_{k}^{\prime}(t)+k^{2} \alpha_{k}(t)\right] \sin (k \xi)=\sum_{k=1}^{\infty} \lambda_{k}(t) \sin (k \xi) \\
\alpha_{1}(0)=\rho \sqrt{\frac{2}{\pi}}, \quad \alpha_{k}(0)=0, \forall k>1 .
\end{array}
$$

We note that $u(t, \cdot) \in B_{1}$ if and only if

$$
\sum_{k=1}^{\infty}\left|\lambda_{k}(t)\right|^{2} \leq \frac{2}{\pi}
$$

Then, for $k>1$, we can keep $\alpha_{k}=0$ taking $\lambda_{k}=0$. Therefore, the optimal strategy is given by

$$
\bar{u}_{1}(t, \xi)=-\sqrt{\frac{2}{\pi}} \sin \xi
$$

ESAIM: Proc., Vol. 4, 1998, 59-72 
and

$$
\bar{y}_{1}(t, \xi)=\left[e^{-t}(1+\rho)-1\right] \sqrt{\frac{2}{\pi}} \sin \xi==2 E
$$

is the corresponding trajectory, obtained solving (21)-(22). From the terminal condition $y\left(t_{0}, \cdot\right) \in \partial B_{r}$ we obtain

$$
e^{-t_{0}}(1+\rho)-1=r
$$

and so

$$
t_{0}=\log \frac{1+\rho}{1+r} .
$$

Next, we show that $T\left(x^{\rho}\right)<t_{0}\left(x^{\rho}\right)$, i.e. that the time needed to reach the target is reduced if we drop condition (20). Let us choose, in (17),

$$
u_{2}(t, \xi)=-\frac{\sin \xi}{\sqrt{\pi}}, \quad t>0, \xi \in(0, \pi) .
$$

Solving the Cauchy-Dirichlet problem

$$
\begin{cases}\frac{\partial y_{2}}{\partial t}-\frac{\partial^{2} y_{2}}{\partial \xi^{2}}=u_{2} & \\ y_{2}(t, 0)=y_{2}(t, \pi)=0, & t>0 \\ y_{2}(0, \xi)=0, & \xi \in(0, \pi)\end{cases}
$$

by Fourier series expansion, we obtain

$$
y_{2}(t, \xi)=-\left(1-e^{-t}\right) \frac{\sin \xi}{\sqrt{\pi}}
$$

Now, let

$$
u_{1}(t, \xi)=-\frac{\sin \xi}{\sqrt{\pi}}
$$

and note that $u=\left(u_{1}, u_{2}\right)$, given by $(26)-(24)$, is an admissible control for our system. Therefore, plugging (25) in (17), we can compute $y_{1}$ :

$$
y_{1}(t, \xi)=\left[(\rho \sqrt{2}+2+t) e^{-t}-2\right] \frac{\sin \xi}{\sqrt{\pi}} .
$$

We now claim that, if $\rho$ is sufficiently large, then

$$
\int_{0}^{\pi}\left(\left|y_{1}\left(t_{0}, \xi\right)\right|^{2}+\left|y_{2}\left(t_{0}, \xi\right)\right|^{2}\right) d \xi<r^{2}
$$

which yields in turn $T\left(x^{\rho}\right)<t_{0}\left(x^{\rho}\right)$. Indeed, (27) is equivalent to

$$
\left(\frac{\rho-r}{1+\rho}\right)^{2}+\left[\left(\rho \sqrt{2}+2+\log \frac{1+\rho}{1+r}\right) \frac{1+r}{1+\rho}-2\right]^{2}<2 r^{2} .
$$


Denoting by $\psi(\rho)$ the left hand side of the above inequality we have that

$$
\lim _{\rho \rightarrow+\infty} \psi(\rho)=1+[(1+r) \sqrt{2}-2]^{2} .
$$

Then we obtain the desired conclusion (27) provided that

$$
r>\frac{3 \sqrt{2}-1}{4} .
$$

In sum, we have proved that, if $\rho$ is sufficiently large, say $\rho>\rho_{0}$, and $y^{*}=\left(y_{1}^{*}, y_{2}^{*}\right)$ is an optimal trajectory for $(17)$ at $x^{\rho}$, then $y_{2}^{*}(t) \neq 0$ for some $t>0$. Being the system symmetric with respect to the map $\left(x_{1}, x_{2}\right) \mapsto\left(x_{1},-x_{2}\right)$, we conclude that another time-optimal trajectory at $x^{\rho}$ is given by

$$
y_{*}(t, \xi)=\left(y_{1}^{*}(t, \xi),-y_{2}^{*}(t, \xi)\right) .
$$

Using a smooth approximation of the absolute value, we provide below an example of a system satisfying (2) and still possessing multiple time-optimal trajectories.

Example 3.2 Let $R=1, r$ and $\rho$ be as in Example 3.1. We define $\left\{\eta_{\epsilon}\right\}_{\epsilon>0}$ to be the uniform approximation of the absolute value given by

$$
\eta_{\epsilon}(s)= \begin{cases}0, & \text { if }|s|<\epsilon, \\ \frac{1}{2}(|s|-\epsilon)^{2}, & \text { if } \epsilon \leq|s|<2 \epsilon, \\ |s|-2 \epsilon+\frac{1}{2} \epsilon^{2}, & \text { if } 2 \epsilon \leq|s| .\end{cases}
$$

Notice that

$$
0 \leq|s|-\eta_{\epsilon}(s) \leq 2 \epsilon, \quad \forall s \in \mathbb{R}, \forall \epsilon>0 .
$$

Now, consider the system

$$
\left\{\begin{array}{l}
\frac{\partial y_{1}}{\partial t}-\frac{\partial^{2} y_{1}}{\partial \xi^{2}}=-\eta_{\epsilon}\left(y_{2}\right)+u_{1} \\
\frac{\partial y_{2}}{\partial t}-\frac{\partial^{2} y_{2}}{\partial \xi^{2}}=u_{2}
\end{array}\right.
$$

under the boundary condition (18). We note that $\eta_{\epsilon}$ is a $C^{1}$ function satisfying (2) with $L_{0}=1$ for every $\epsilon>0$. Moreover, $\omega=1$ as before, and so condition (7) is also satisfied.

We want to show that, for some $\epsilon>0$, if we choose the same initial condition $x^{\rho}$ as in (19), then the minimum time problem has multiple optimal trajectories. Consequently, the corresponding minimum time function, $T^{\epsilon}$, is singular at $x^{\rho}$ by Theorem 2.3.

ESAIM: Proc., Vol. 4, 1998, 59-72 
Following the same reasoning as in the previous example, it suffices to show that

$$
T^{\epsilon}\left(x^{\rho}\right)<t_{0}^{\epsilon}\left(x^{\rho}\right),
$$

where $t_{0}^{\epsilon}$ is the minimum time needed to steer system (30) to $B_{r}$ under the state constraint $y_{2} \equiv 0$. $=$ We note that

$$
t_{0}^{\epsilon}\left(x^{\rho}\right)=t_{0}\left(x^{\rho}\right)
$$

for all $\epsilon>0$, with $t_{0}$ given by (23).

Let $u=\left(u_{1}, u_{2}\right)$ be defined as in (26), (24), and let $y$ and $y^{\epsilon}$ denote the solutions of problems (17), (18) and (30), (18), respectively, with initial condition $x^{\rho}$. In order to obtain (31), we shall prove that

$$
\left\|y^{\epsilon}\left(t_{0}\right)\right\|<r .
$$

To this end, observe that $y_{2}^{\epsilon}=y_{2}$, and

$$
\frac{\partial}{\partial t}\left(y_{1}^{\epsilon}-y_{1}\right)-\frac{\partial^{2}}{\partial \xi^{2}}\left(y_{1}^{\epsilon}-y_{1}\right)=\left|y_{2}\right|-\eta_{\epsilon}\left(y_{2}\right) .
$$

Hence, using estimate (29) and the Poincaré inequality, we conclude that

$$
\begin{gathered}
\frac{1}{2} \frac{d}{d t}\left\|y_{1}^{\epsilon}-y_{1}\right\|^{2}+\left\|\frac{\partial}{\partial \xi}\left(y_{1}^{\epsilon}-y_{1}\right)\right\|^{2}=\int_{0}^{\pi}\left(\left|y_{2}\right|-\eta_{\epsilon}\left(y_{2}\right)\right)\left(y_{1}^{\epsilon}-y_{1}\right) d \xi \\
\leq \frac{1}{4} \int_{0}^{\pi}\left(\left|y_{2}\right|-\eta_{\epsilon}\left(y_{2}\right)\right)^{2} d \xi+\left\|y_{1}^{\epsilon}-y_{1}\right\|^{2} \leq \pi \epsilon^{2}+\left\|\frac{\partial}{\partial \xi}\left(y_{1}^{\epsilon}-y_{1}\right)\right\|^{2}
\end{gathered}
$$

Thus,

$$
\left\|y_{1}^{\epsilon}\left(t_{0}\right)-y_{1}\left(t_{0}\right)\right\| \leq \epsilon \sqrt{2 \pi t_{0}} .
$$

In view of (27), the last inequality yields (32) if $\epsilon$ is sufficiently small.

\section{References}

[1] Agmon S., Douglis A.,Nirenberg L., Estimates near the boundary for solutions of elliptic partial differential equations satisfying general boundary conditions, Comm. Pure Appl. Math. 12 (1959), 623-727.

[2] Albano P., Cannarsa P., Sinestrari C., Singularities of Semi-Concave Functions in Infinite Dimensions. (in preparation)

[3] Balakrishnan A.V., Optimal control problems in Banach spaces, SIAM J. Control 3 (1965), 152-180.

[4] Barbu V., The dynamic programming equation for the time-optimal control problem in infinite dimensions, SIAM J. Control Optim. 29 (1991), 445-456. 
[5] Barbu V., Analysis and control of nonlinear infinite dimensional systems, Academic Press, Boston, 1993.

[6] Bensoussan A., Da Prato G., Delfour M.C., Mitter S.K., Represantation and control of infinite dimensional systems, Birkhäuser, Boston, 1992.

[7] Cannarsa P., Frankowska H., Value functions and optimality conditions for semilinear control problems, Appl. Math. Optim. 26 (1992), $139-169$.

[8] Cannarsa P., Frankowska H., Value functions and optimality conditions for semilinear control problems II: parabolic case, Appl. Math. Optim. 33 (1996), 1-33.

[9] Cannarsa P., Sinestrari C., An infinite dimensional time optimal control problem

[10] Cannarsa P., Terreni B., Vespri V., Analytic semigroups generated by non variational elliptic systems of second order under Dirichl= et boundary conditions, J. Math. Anal. Appl. 112 (1985), 56-103.

[11] Cannarsa P., Tessitore M.E., Optimality conditions for boundary control problems of parabolic type, International Series of Numerical Mathematics, Vol. 118, Birkhäuser, Basel, 1994, 79-96.

[12] Cârja O., On the minimal time function for distributed control systems in Banach spaces, J. Optim. Theory Appl. 44 (1984), 397-406.

[13] Cârja O., On continuity of the minimal time function for distributed control systems, Boll. Un. Mat. Ital. 6 (1985), 293-302.

[14] Conti R., Time-optimal solution of a linear evolution equation in Banach spaces, J. Optim. Theory Appl. 2 (1968), 277-284.

[15] Egorov Yu. V., Optimal control in Banach spaces, Dokl. Acad. Nauk SSSR 150 (1963), 241-244 (in Russian).

[16] Egorov Yu. V., Certain problems in optimal control theory, USSR Comput. Math. \& Phis. 3 (1963), 1209-1232 (in Russian).

[17] Fattorini H.O., Time-optimal control of solutions of operational differential equations, SIAM J. Control 2 (1964), 54-59.

[18] Fattorini H.O., The time-optimal control problems in Banach spaces, Appl. Math. Optim. 1 (1974), 163-188.

[19] Friedman A., Optimal control in Banach spaces with fixed endpoints, J. Math. Anal. Appl. 24 (1968), 161-181. 
[20] Li X., Yong J., Optimal control theory for infinite dimensional systems, Birkhäuser, Boston, 1995.

[21] Lions J.L., Optimal control of systems governed by partial differential equations, Springer-Verlag, Berlin, 1971.

[22] Lunardi A., Analytic semigroups and optimal regularity in parabolic problems, Birkhäuser, Berlin, 1995. 